\title{
基于磷酸酯基的醇溶性六芴基苯的合成及其 在倒置聚合物太阳能电池中的应用
}

刘源 ${ }^{(1)}$, 张文俊 ${ }^{2}$ ，方俊锋 ${ }^{(2 *}$, 杨楚罗 $^{(1) *}$

(1) 有机化工新材料湖北省协同创新中心; 湖北省有机高分子光电功能材料重点实验室; 武汉大学化学与分子科学学院, 武汉 430072

(2) 中国科学院宁波材料技术与工程研究所, 宁波 315201

*通讯作者, E-mail: fangjf@nimte.ac.cn; clyang@whu.edu.cn

收稿日期: 2015-12-15; 接受日期: 2016-01-19; 网络版发表日期: 2016-04-19

国家重点基础研究发展计划(编号: 2013CB834805)和国家自然科学基金(编号: 21572171, 51125013)资助项目

摘要 本文报道了两个基于六芴基苯为中心核的星型分子 $\mathrm{Tp} 0$ 和 $\mathrm{Tp} 1$. 将磷酸酯基团引入芴的侧链, 保证了分 子的醇溶性, 同时由于磷酸酯极性基团形成界面偶极矩, 极大地降低了 ITO电极的功函数. 将 $\mathrm{Tp} 0$ 和 $\mathrm{Tp} 1$ 分子应 用于倒置聚合物太阳能器件, 与无界面材料的器件相比提升了开路电压 $\left(V_{\mathrm{oc}}\right)$ 和填充因子 $(\mathrm{FF})$, 基于 $\mathrm{Tp} 0$ 和 $\mathrm{Tp} 1$ 为 界面材料的器件分别实现了 $5.30 \%$ 和 $5.12 \%$ 的光电转换效率.

关键词磷酸酯基, 醇溶性, 界面材料, 聚合物太阳能电池

\section{1 引言}

有机聚合物太阳能电池因其柔性、低成本、可通 过溶液加工或打印制备大面积器件的特性而引起人 们的广泛关注 ${ }^{[1,2]}$. 近几年来, 聚合物太阳能电池的 器件效率迅速提高, 单结聚合物太阳能电池的能量 转换效率(PCE) 已经超过 $10 \%{ }^{[3,4]}$. 如何进一步提升器 件性能成为当下的研究热点.

除了聚合物材料性能的改善, 器件结构的优化 也是提升 PCE 的重要方式 ${ }^{[5,6]}$. 传统的正置器件结构 通常使用具有腐蚀性的 PEDOT:PSS 作为空穴传输层 材料, 并使用易氧化的钡、钙等低功函数材料作为阴 极, 从而影响器件的空气稳定性 ${ }^{[7]}$. 倒置器件结构除
了避免使用这些材料外, 还可利用本体异质结两相 材料的浓度垂直分布, 实现更有效的电子提取 ${ }^{[8,9]}$. 然而, 氧化铟锡(ITO) 并不是一个理想的倒置器件的 阴极, 其功函数与常用的受体材料 PCBM 的最低未 占轨道能级 (LUMO) 并不匹配, 阻碍了电子的提取. 在 ITO 电极与活性层之间插入界面修饰材料是一种 有效的降低 ITO 电极功函数的方法, 修饰后的 ITO更 适合作为倒置器件的阴极 ${ }^{[10,11]}$.

近几年来，诸多应用于聚合物太阳能电池的界 面材料已被报道 ${ }^{[12,13]}$, 其类型多种多样，包括金属 氧化物 ${ }^{[14]}$ 、碱金属化合物 ${ }^{[15]}$ 、富勒烯衍生物 ${ }^{[16]}$ 、自 组装单分子层 $(\mathrm{SAM})^{[17]}$ 和醇溶性共轭或非共轭聚合 物 $^{[4]}$. 其中, 曹锯课题组 ${ }^{[18,19]}$ 报道了一系列基于醇溶

\footnotetext{
引用格式: 刘源, 张文俊, 方俊锋, 杨楚罗. 基于磷酸酯基的醇溶性六荡基苯的合成及其在倒置聚合物太阳能电池中的应用. 中国科学: 化学, 2016, 46: 510-516

Liu Y, Zhang WJ, Fang JF, Yang CL. Synthesis of alcohol soluble hexakis(fluoren-2-yl)benzene based on phosphate groups and their application in inverted polymer solar cells. Sci Sin Chim, 2016, 46: 510-516, doi: 10.1360/N032015-00263
} 
性共轭聚芴的界面材料, 并实现了优良的器件性能. 某些特定的功能基团, 如氨基、铵盐基、二乙醇胺基、 磷酸酯基和氧化氨基等极性基团被接入共轭聚芴的 侧链, 使得这些聚合物具有水/醇溶性 ${ }^{[20,21]}$. 此外, 由 于选择正交溶剂, 避免了界面层在器件制备过程中 被上层旋涂的活性层破坏. 进一步的研究表明, 这些 含有极性基团的界面材料在电极表面产生界面偶极 矩, 降低了电极的功函数, 从而减小甚至消除了电极 和活性层之间的接触势垒, 提高了电极对电荷的收 集效率 ${ }^{[7]}$. 最近, 一些具有确定化学结构的小分子界 面材料也被用于倒置聚合物太阳能电池, 同样取得 了良好的器件效果 ${ }^{[22,23]}$. 与聚合物界面材料相比, 理 论上小分子界面材料具有更好的重现性, 不受分子 量和分散度(PDI)影响.

具有 $\pi$ 共轭结构的小分子材料, 可以通过调节共 轭长度改变分子性质, 有助于我们更好地理解结构 性能关系. 本课题组 ${ }^{[24,25]}$ 之前的研究表明, 基于六夰 基苯的分子显示出优异的性质. 六芴基苯是一类具 有星型 $\pi$ 共轭结构的寡聚物, 6 个芴单元通过 2 位连 接于中心苯环, 同时芴的 9 位引入长烷基链, 使得这 类分子具有良好的溶解性、成膜性能以及高热稳定性. 最近, 我们在荡的侧链末端引入氮羟乙基, 将其作为 修饰 ITO 电极的界面材料, 取得了良好的器件性 能 ${ }^{[26]}$. 然而, 不同极性基团的作用效果在文献中鲜有 报道. 本文将磷酸酯基极性基团引入星型六芴基苯 结构中, 合成了两个醇溶性界面材料 $\mathrm{Tp} 0$ 和 $\mathrm{Tp} 1$ (图 1 ), 测试了分子的光物理化学性质和电化学性质, 并 初步表征了其在采用 PTB7 和 $\mathrm{PC}_{71} \mathrm{BM}$ 为活性层材料 的倒置太阳能电池中的性能.

\section{2 实验部分}

\section{1 试剂与测试仪器}

实验中合成所需的亚磷酸三乙酯购自 Alfa 公司 (中国). 核磁共振谱采用 Varian MERCURY-VX300 核磁共振仪(美国); 飞行时间质谱(MALDI-TOF)采用 Bruker BIFLEX III TOF 质谱仪(德国); 紫外-可见光 谱采用 Shimadzu UV-2550 型紫外-可见光谱仪(日本); 循环伏安法采用 CHI600A 电化学工作站, 工作电极 为铂电极, 参比电极为银/氯化银 $(\mathrm{Ag} / \mathrm{AgCl})$ 电极, 对 电极为铂丝, 二茂铁为内标, 电解质为 $0.1 \mathrm{~mol} \mathrm{~L}^{-1}$ 四 正丁基铵六氟磷酸盐 $\left(n-\mathrm{Bu}_{4} \mathrm{NPF}_{6}\right)$ 乙腈溶液.

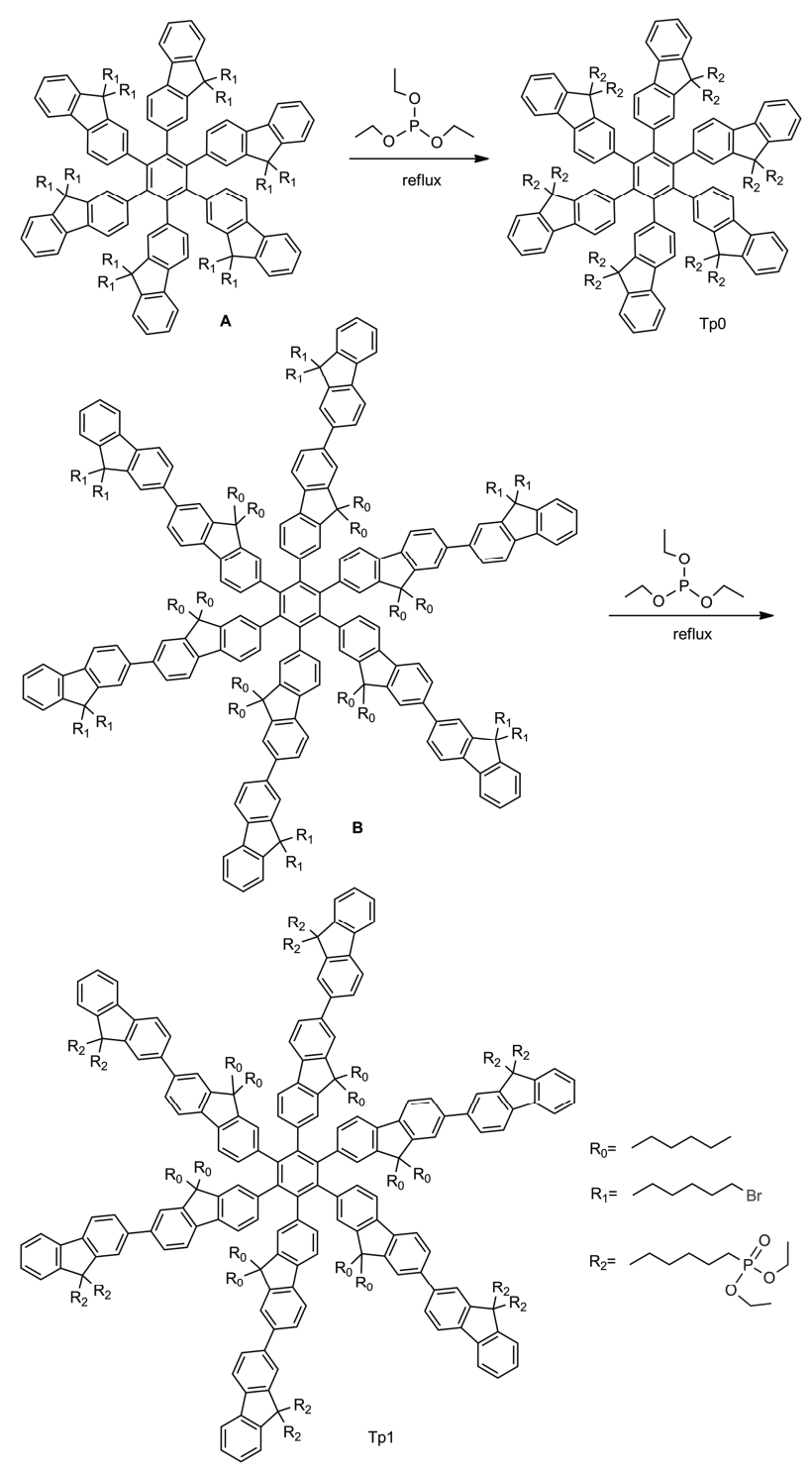

图 1 界面材料 $\mathrm{Tp} 0$ 和 $\mathrm{Tp} 1$ 的分子结构与合成路线

\section{2 器件制备}

ITO 玻璃祄底用专用清洗液超声清洗后再分别 经去离子水、丙酮、异丙醇清洗数次, 氮气吹干后再 紫外臭氧处理. 配制不同浓度的界面材料 $\mathrm{Tp} 0$ 和 $\mathrm{Tp} 1$ 的甲醇溶液 $\left(0.1 \sim 1 \mathrm{mg} \mathrm{mL} \mathrm{m}^{-1}\right)$ 旋涂于 ITO 玻璃祄底. $\mathrm{PTB} 7 / \mathrm{PC}_{71} \mathrm{BM}$ 活性层材料(10:15, 质量比)溶于氯苯/ 1,8-二碘辛烷 (97:3, 体积比), 旋涂于界面层上, 厚度 约 $90 \pm 10 \mathrm{~nm}$, 最后真空下蒸镀三氧化钿空穴传输层 和铝电极, 器件的有效面积约为 $0.06 \mathrm{~cm}^{2}$. 电流密度电压 $(J-V)$ 特征曲线采用 Keithley 236 测量表(美国); 
PCE 在 AM $1.5 \mathrm{G}$ 光源模拟器条件下测量; 紫外光电 子能谱(UPS)采用 Kratos AXIS ULTRA DLD 能谱仪 (日本); 薄膜厚度测量采用 Veeco Dektak150 台阶仪 (美国); 原子力显微镜 (AFM) 采用 Shimadzu SPM9700 扫描探针显微镜 (日本); 接触角测试采用 Dataphysics OCA20 光学成像仪(德国).

\section{3 界面材料的合成}

两个界面材料的中间体 A、B 参考文献[26]的方 法合成.

1,2,3,4,5,6-六(9,9-双(6-(二乙基磷酸酯)正己基)芴)苯 (Tp0): 在 $25 \mathrm{~mL}$ 圆底烧瓶中加入 $0.3 \mathrm{~g}$ 1,2,3,4,5,6-六 $(9,9$-双(6-溴正已基)-芴)苯(A)和 $5 \mathrm{~mL}$ 亚 磷酸三乙酯, 于 $150^{\circ} \mathrm{C}$ 加热回流 $48 \mathrm{~h}$ 后, 蒸除多余溶 剂, 粗产品以正己烷/甲醇/三乙胺(100:10:2, 体积比) 为淋洗剂进行柱层析, 得到淡黄色固体, 产率 $75 \%$. ${ }^{1} \mathrm{H}$ NMR (300 MHz, $\left.\mathrm{CD}_{3} \mathrm{OD}, \delta\right): 7.41 \sim 6.98(\mathrm{~m}, 42 \mathrm{H})$, $4.10(\mathrm{~s}, 72 \mathrm{H}), 2.20 \sim 2.15(\mathrm{~m}, 24 \mathrm{H}), 1.84(\mathrm{br}, 24 \mathrm{H})$, 1.29 1.19 (m, 48H), 1.04 0.99 (m, 96H). ${ }^{13} \mathrm{C}$ NMR $(75$ $\mathrm{MHz}, \mathrm{CD}_{3} \mathrm{OD}, \delta$ ): 152.79, 150.97, 150.02, 142.60, $141.94,138.71,130.93,127.95,127.22,123.92,121.73$, 119.20, 55.88, 53.22, 40.61, 33.23, 31.19, 28.23, 27.07, 24.70, 24.34, 22.33. MS (MALDI-TOF, $m / z): 3704.1$.

1,2,3,4,5,6-六 $\left(9^{\prime}, 9^{\prime}\right.$-双(6-(二乙基磷酸酯)正己基9,9-二正己基-二联芴)苯( $\mathrm{Tp} 1)$ : $\mathrm{Tp} 1$ 的合成方法参照 $\mathrm{Tp} 0$ 的合成. 粗产品以正己烷/甲醇/三乙胺(100:10:3, 体积比)为淋洗剂进行柱层析, 得到淡黄色固体, 产 率 70\%. ${ }^{1} \mathrm{H}$ NMR $\left(300 \mathrm{MHz}, \mathrm{CD}_{3} \mathrm{OD}, \delta\right): 7.79 \sim 7.71(\mathrm{~m}$, 24H), 7.63 7.51 (m, 36H), 7.39 7.30 (m, 18H), $4.11(\mathrm{~s}$, $72 \mathrm{H}), 2.43 \sim 2.40(\mathrm{~m}, 48 \mathrm{H}), 2.02(\mathrm{br}, 24 \mathrm{H}), 1.29 \sim 1.20$ (m, 144H), 1.04 0.99 (m, 96H), 0.96 (br, 36H). ${ }^{13} \mathrm{C}$ NMR $\left(75 \mathrm{MHz}, \mathrm{CD}_{3} \mathrm{OD}, \delta\right)$ : 153.79, 152.51, 151.83, 151.01, 142.36, 142.02, 141.96, 141.16, 138.82, 134.86, $128.33,126.73,124.21,122.52,121.75,56.83,56.17$, 53.35, 41.90, 41.26, 33.70, 33.01, 32.02, 31.07, 30.38, $28.31,26.16,25.38,24.73,24.12,15.19,14.89$. MS (MALDI-TOF, $\mathrm{m} / \mathrm{z}$ ): 5699.2.

\section{3 结果与讨论}

\section{1 光物理化学性质和电化学性质}

图 2(a) 是 $\mathrm{Tp} 0$ 和 $\mathrm{Tp} 1$ 在甲醇溶液 $\left(10^{-6} \mathrm{~mol} \mathrm{~L}^{-1}\right)$ 和薄膜中的紫外可见光吸收光谱, 均表现出强烈的 $\pi-\pi *$ 吸收带, 并且随着共轭长度的增加, 在甲醇溶液 中的最大吸收波长从 $314 \mathrm{~nm}$ 红移到 $339 \mathrm{~nm}$. 两者在 薄膜中的最大吸收波长分别为 316 和 $340 \mathrm{~nm}$, 与甲 醇溶液相比几乎没有变化. 与之前的研究对比可以 发现, 磷酸酯基团连接到非共轭的长烷基链上或醇 溶性基团的改变, 对六芴基苯的光物理化学性质几 乎没有影响，仍然是由其基本骨架决定的 ${ }^{[24]}$. 同时, 两个化合物在可见光区和近红外区均没有吸收，在 用作界面材料时，可以保证光的高透过率. 由于六芴 基苯中心核具有高度扭曲的结构，因此 $\mathrm{Tp} 0$ 和 $\mathrm{Tp} 1$ 均 表现出了较高的光学带隙. 根据分子能隙的计算公 式 $E_{\mathrm{g}}=1240 / \lambda$ (其中 $\lambda$ 为紫外吸收光谱起始位置对应的 波长), 计算得出 $\mathrm{Tp} 0$ 和 $\mathrm{Tp} 1$ 的光学带隙分别为 3.63 和 $3.25 \mathrm{eV}$.

应用循环伏安法测量了 $\mathrm{Tp} 0$ 和 $\mathrm{Tp} 1$ 的电化学性 质, 如图 2(b)所示. 根据 Leeuw 经验公式进行计算, 以二茂铁为内标 (二茂铁对应的真空能级为 $-4.8 \mathrm{eV}$ ),
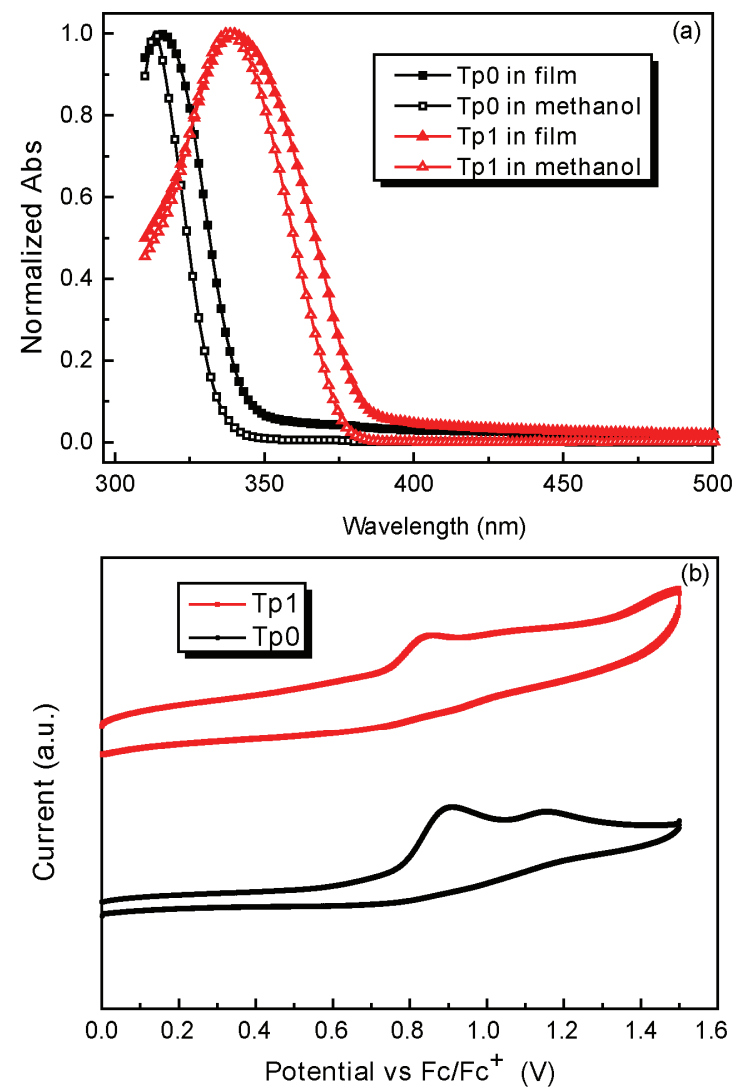

图 2 (a) 归一化的 $\mathrm{Tp} 0$ 和 $\mathrm{Tp} 1$ 在甲醇溶液和薄膜中的紫外 可见光吸收光谱图; (b) $\mathrm{Tp} 0$ 和 $\mathrm{Tp} 1$ 在乙腈溶液中的循环伏安 曲线(网络版彩图) 
其最高占据轨道能级 $(\mathrm{HOMO})$ 为 $\mathrm{HOMO}=-\left(\left[E_{\mathrm{onset}}\right]^{\mathrm{ox}}+\right.$ 4.8) $\mathrm{eV}$, 通过氧化电位起始位置, 计算得到其 HOMO 能级分别为 -5.56 和 $-5.58 \mathrm{eV}$, 通过其光学带 隙计算, 得到两者的 LUMO 能级分别为-1.93 和 $-2.33 \mathrm{eV} . \mathrm{Tp} 0$ 和 $\mathrm{Tp} 1$ 的光物理化学性质和电化学性 质的总结见表 1 .

\section{2 紫外光电子能谱分析}

采用紫外光电子能谱(UPS)测量了旋涂 $\mathrm{Tp} 0$ 和 $\mathrm{Tp} 1$ 的 ITO 表面功函数, 如图 3 所示, 当无界面材料 覆盖时, ITO 功函数为 $-4.41 \mathrm{eV}$, 而分别旋涂两个界 面材料后的 ITO 其二次电子截止都移向更高的束缚 能, 表明其修饰后的表面功函数显著下降, 计算得到

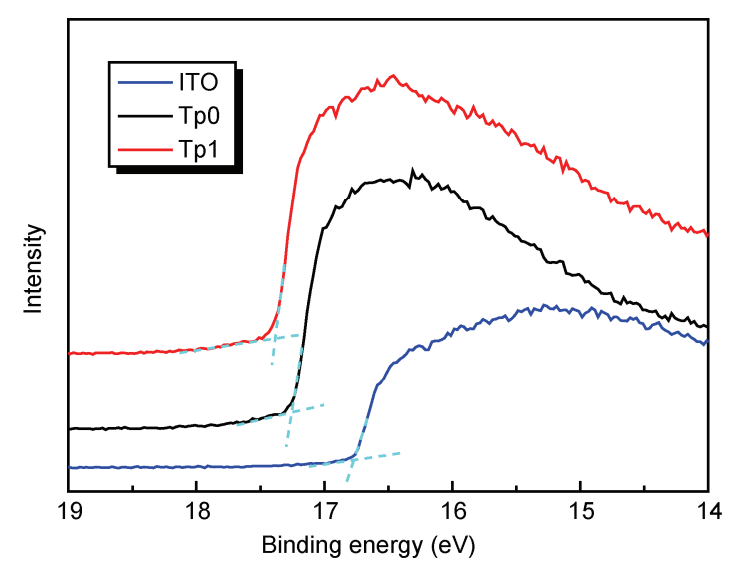

图 3 ITO 和旋涂 Tp0 和 Tp1 后紫外光电子能谱示意图(网 络版彩图)
其功函数分别为 -3.95 和 $-3.79 \mathrm{eV}$. 含有磷酸酯基团 的界面材料与 ITO 表面接触时, 生成界面偶极矩, 降 低了电极功函数. 而 $\mathrm{Tp} 0$ 和 $\mathrm{Tp} 1$ 修饰后的功函数不同, 可能在于其不同的分子结构和堆积方式. 降低了功 函数的 ITO 电极与活性层中的富勒烯受体材料形成 欧姆接触, 有利于电子从活性层到电极的传输, 从而 提升聚合物太阳能电池的器件性能 ${ }^{[27]}$.

\section{3 器件性能}

紫外光电子能谱分析显示出 $\mathrm{Tp} 0$ 和 $\mathrm{Tp} 1$ 作为 ITO 电极界面修饰的应用潜力. 为进一步探究其实际作 用效果, 我们制备了倒置的聚合物太阳能电池器件, $\mathrm{PTB} 7$ 和 $\mathrm{PC}_{71} \mathrm{BM}$ 作为活性层的给受体材料, 其器件 结构为 $\mathrm{ITO} /$ 界面/PTB7: $\mathrm{PC}_{71} \mathrm{BM} /$ 三氧化钼/铝. 在 $\mathrm{AM}$ $1.5 \mathrm{G} 100 \mathrm{~mW} \mathrm{~cm}$ 光源条件下的 $J-V$ 特征曲线如图 4 所示, 详细的器件性能参数见表 2 .

之前的研究表明, 由于此类界面材料的迁移率 和导电性较低, 其薄膜厚度显著影响器件效果. 我们 首先优化了界面层的厚度, 在最佳的厚度条件下, 基 于 Tp0 和 Tp1 界面的器件的 PCE 分别达到了 $5.30 \%$ 和 $5.12 \%$, 与无界面修饰的器件相比, 其开路电压 $\left(V_{\mathrm{oc}}\right)$ 和填充因子 $(\mathrm{FF})$ 显著增大, 表明在界面材料的作 用下, 由于电极表面功函数的降低, 电子从活性层向 电极的传递势垒明显降低, 从而导致器件性能的提 升. 然而与之前基于氮羟乙基界面材料的器件相比, 其 $V_{\mathrm{oc}}$ 和 $\mathrm{FF}$ 又较低, 器件的串联电阻显著增大. 为探 究其原因, 我们测试了其表面润湿性和形貌.

表 $1 \mathrm{Tp} 0$ 和 $\mathrm{Tp} 1$ 的紫外可见光吸收光谱和电化学数据

\begin{tabular}{ccccccc}
\hline Molecule & $\lambda_{\text {abs }}(\mathrm{nm})^{\mathrm{a})}$ & $\lambda_{\text {abs }}$, film $(\mathrm{nm})$ & $E_{\mathrm{g}}{ }^{\text {opt }}(\mathrm{eV})^{\mathrm{b})}$ & $E_{\text {ox }}(\mathrm{V})^{\mathrm{c})}$ & HOMO $(\mathrm{eV})^{\mathrm{d})}$ & LUMO $(\mathrm{eV})$ \\
\hline $\mathrm{Tp} 0$ & 314 & 316 & 3.63 & 0.76 & -5.56 & -1.93 \\
$\mathrm{Tp} 1$ & 339 & 340 & 3.25 & 0.78 & -5.58 & -2.33 \\
\hline
\end{tabular}

a) $10^{-6} \mathrm{~mol} \mathrm{~L}^{-1}$ 的甲醇溶液; b) 通过薄膜中的紫外可见光吸收光谱起始位置计算; c) 扫描速度为 $100 \mathrm{mV} \mathrm{s}^{-1}$, 二茂铁在乙腈溶液中对应 $\mathrm{Ag} / \mathrm{AgCl}$ 参比电极氧化电位为 $-0.53 \mathrm{~V} ; \mathrm{d}$ ) 通过氧化电位起始位置计算

表 2 基于 $\mathrm{Tp} 0$ 和 $\mathrm{Tp} 1$ 界面材料的器件性能参数

\begin{tabular}{cccccc}
\hline Interlayer & $V_{\mathrm{oc}}(\mathrm{V})$ & $J_{\mathrm{sc}}\left(\mathrm{mA} \mathrm{cm}^{-2}\right)^{\mathrm{a})}$ & $\mathrm{FF}(\%)$ & $\mathrm{PCE}(\%)$ & $R_{\mathrm{s}}\left(\Omega \mathrm{cm}^{-2}\right)^{\mathrm{b})}$ \\
\hline Bare ITO & 0.25 & 14.02 & 33.46 & 5.18 \\
Tp0 & 0.61 & 15.81 & 54.56 & 5.30 \\
Tp1 & 0.62 & 15.73 & 52.26 & 5.12 \\
\hline
\end{tabular}

a) 短路电流; b) 串联电阻 


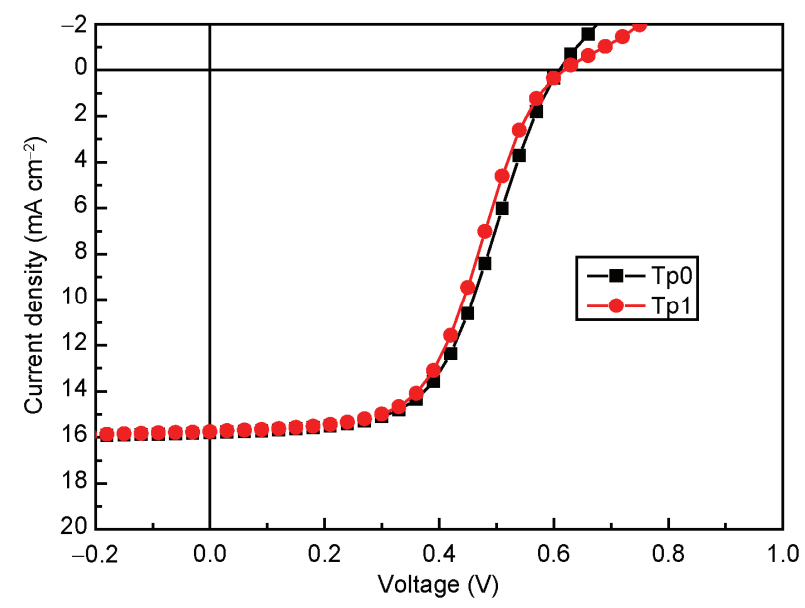

图 $4 \mathrm{Tp} 0$ 和 $\mathrm{Tp} 1$ 作为界面材料的器件 $J-V$ 特征曲线

\section{4 界面润湿性与形貌}

通过测量接触角进一步研究了界面材料的润湿 性能, 水滴实验的照片如图 5 所示. $\mathrm{Tp} 0$ 的界面亲疏 水性与无修饰的 ITO 电极相当, 分别为 $24.5^{\circ}$ 和 $29.2^{\circ}$. 然而, Tp1 的界面表现出了明显增强的疏水性, 其接 触角增加到 $44.8^{\circ}$, 这与其分子结构的变化一致. 与 $\mathrm{Tp} 0$ 相比, $\mathrm{Tp} 1$ 的 $\pi$ 电子更加离域，同时疏水性的正己 基被引入到内侧的芴单元. 接触角的变化趋势与基 于氮羟乙基的界面材料研究结果一致. 由于磷酸酯 基的极性比氮羟乙基的极性更大，因此基于磷酸酯 基的化合物比基于氮羟乙基的化合物的水/醇溶性更 好, 其界面接触角也明显更小.

由于旋涂界面所用溶剂甲醇与旋涂活性层所用 溶剂氯苯为正交溶剂, 旋涂活性层并不会破坏下层 的界面材料. 为进一步找出器件性能提升效果不明 (a)

(b)

$24.5^{\circ}$

$29.2^{\circ}$
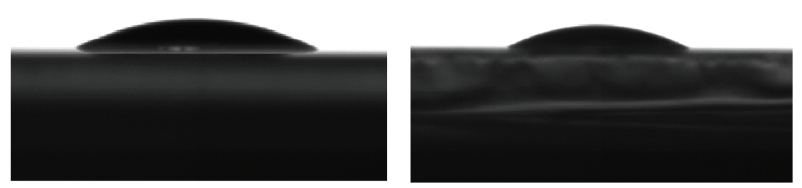

(c)

$44.8^{\circ}$

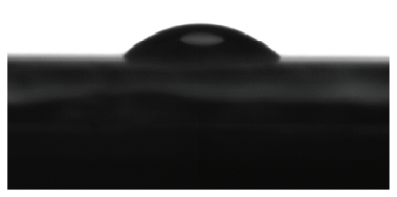

图 $5 \mathrm{ITO}(\mathrm{a})$ 和旋涂 $\mathrm{Tp} 0(\mathrm{~b})$ 和 $\mathrm{Tp} 1$ 后(c)的界面接触角

显的原因, 我们应用原子力显微镜探针测量了界面 材料的表面形貌. 如图 6 所示, 两个材料旋涂到 ITO 后的表面都比较粗䊁, 其粗粘度均方根 $(\mathrm{rms})$ 分别为 3.72 和 $3.88 \mathrm{~nm}$. 在原子力显微镜的三维高度图上可 观察到少量针孔(pin hole), 这些针孔的存在使得活 性层与电极直接接触产生漏电流, 影响器件短路电 流的提升. 此外, 由于界面层的薄膜形貌粗粘, 使得 ITO 电极与活性层的接触较差, 器件的串联电阻较大. 由于较大的串联电阻的影响, 基于 $\mathrm{Tp} 0$ 和 $\mathrm{Tp} 1$ 的器件 都有不同程度的电压降，使得 $V_{\mathrm{oc}}$ 较低. 同时较差的 活性层与电极的接触也抑制了载流子的传输, 使得 $\mathrm{FF}$ 也较低 ${ }^{[28]}$. 与之前研究的基于氮羟乙基的界面材 料对比可知, 正是磷酸酯基团的存在, 使得该类化合 物在 ITO 电极上旋涂时得不到理想的薄膜形貌. Tp0 和 $\mathrm{Tp} 1$ 较差的成膜性导致基于该磷酸酯基的界面材 料的器件性能相对较差.
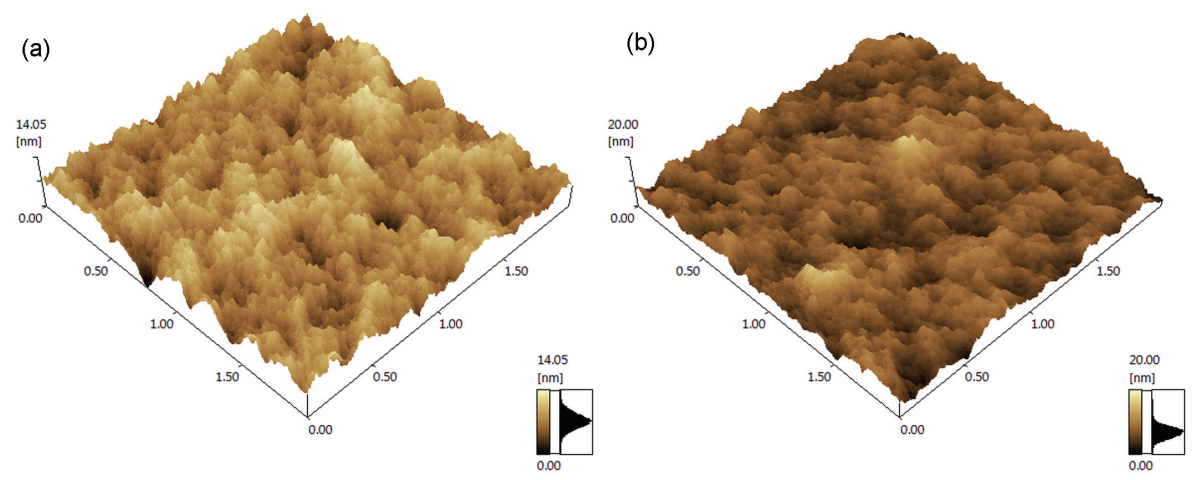

图 $6 \mathrm{Tp} 0$ (a)和 Tp1 (b)旋涂在 ITO 上的原子力显微镜三维高度图(网络版彩图) 


\section{4 结论}

本文合成了两个以六芴基苯为中心核，含有磷 酸酯极性基团的星型分子，其醇溶特性和降低 ITO 电极功函数的能力显示了其在倒置聚合物太阳能电
池中的潜在应用. 基于该两个分子为界面材料的器 件实现了 $5.30 \%$ 和 $5.12 \%$ 的 PCE. 初步研究结果表明, 含有磷酸酯基团的此类分子成膜性不理想, 导致器 件性能无法进一步提升. 未来的工作将围绕极性基 团的替换展开，以期实现更好的器件性能.

\section{参考文献}

1 Thompson BC, Fréchet JMJ. Angew Chem Int Ed, 2008, 47: 58-77

2 Kippelen B, Bredas JL. Energy Environ Sci, 2009, 2: 251-261

3 He Z, Xiao B, Liu F, Wu H, Yang Y, Xiao S, Wang C, Russell TP, Cao Y. Nat Photon, 2015, 9: 174-179

4 Ouyang X, Peng R, Ai L, Zhang X, Ge Z. Nat Photon, 2015, 9: 520-524

5 Chen LM, Hong Z, Li G, Yang Y. Adv Mater, 2009, 21: 1434-1449

6 Kyaw AKK, Wang DH, Gupta V, Zhang J, Chand S, Bazan GC, Heeger AJ. Adv Mater, 2013, 25: 2397-2402

7 He Z, Zhong C, Huang X, Wong WY, Wu H, Chen L, Su S, Cao Y. Adv Mater, 2011, 23: 4636-4643

8 Campoy-Quiles M, Ferenczi T, Agostinelli T, Etchegoin PG, Kim Y, Anthopoulos TD, Stavrinou PN, Bradley DDC, Nelson J. Nat Mater, 2008, 7: 158-164

9 Xu Z, Chen LM, Yang G, Huang CH, Hou J, Wu Y, Li G, Hsu CS, Yang Y. Adv Funct Mater, 2009, 19: 1227-1234

10 Chen LM, Xu Z, Hong Z, Yang Y. J Mater Chem, 2010, 20: 2575-2598

11 Yip HL, Jen AKY. Energy Environ Sci, 2012, 5: 5994-6011

12 He Z, Wu H, Cao Y. Adv Mater, 2014, 26: 1006-1024

13 Huang F, Wu H, Cao Y. Chem Soc Rev, 2010, 39: 2500-2521

14 Chen S, Manders JR, Tsang SW, So F. J Mater Chem, 2012, 22: 24202-24212

15 Liao HH, Chen LM, Xu Z, Li G, Yang Y. Appl Phys Lett, 2008, 92: 173303

16 Page ZA, Liu Y, Duzhko VV, Russell TP, Emrick T. Science, 2014, 346: 441-444

17 Song CK, White AC, Zeng L, Leever BJ, Clark MD, Emery JD, Lou SJ, Timalsina A, Chen LX, Bedzyk MJ, Marks TJ. ACS Appl Mater Interf, 2013, 5: 9224-9240

18 Liu S, Zhang K, Lu J, Zhang J, Yip HL, Huang F, Cao Y. J Am Chem Soc, 2013, 135: 15326-15329

19 He Z, Zhong C, Su S, Xu M, Wu H, Cao Y. Nat Photon, 2012, 6: 591-595

20 Guan X, Zhang K, Huang F, Bazan GC, Cao Y. Adv Funct Mater, 2012, 22: 2846-2854

21 Duan C, Zhang K, Guan X, Zhong C, Xie H, Huang F, Chen J, Peng J, Cao Y. Chem Sci, 2013, 4: 1298-1307

22 Zhang W, Wu Y, Bao Q, Gao F, Fang J. Adv Energy Mater, 2014, 4: 1400359

23 Zhang Q, Zhang D, Li X, Liu X, Zhang W, Han L, Fang J. Chem Commun, 2015, 51: 10182-10185

24 Zou Y, Zou J, Ye T, Li H, Yang C, Wu H, Ma D, Qin J, Cao Y. Adv Funct Mater, 2013, 23: 1781-1788

25 Zou Y, Ye T, Ma D, Qin J, Yang C. J Mater Chem, 2012, 22: 23485-23491

26 Zou Y, He Z, Zhao B, Liu Y, Yang C, Wu H, Cao Y. Sci Rep, 2015, 5: 17329

27 He C, Zhong C, Wu H, Yang R, Yang W, Huang F, Bazan GC, Cao Y. J Mater Chem, 2010, 20: 2617-2622

28 Pang C, Chellappan V, Yim JH, Tan MJ, Goh GTW, Lee S, Zhang J, de Mello J. ACS Appl Mater Interf, 2015, 7: 5219-5225 


\title{
Synthesis of alcohol soluble hexakis(fluoren-2-yl)benzene based on phosphate groups and their application in inverted polymer solar cells
}

\author{
Yuan Liu ${ }^{1}$, Wenjun Zhang ${ }^{2}$, Junfeng Fang ${ }^{2 *}$, Chuluo Yang ${ }^{1 *}$ \\ 1 Hubei Collaborative Innovation Center for Advanced Organic Chemical Materials; Hubei Key Laboratory on Organic and Polymeric \\ Optoelectronic Materials; College of Chemistry and Molecular Sciences, Wuhan University, Wuhan 430072, China \\ 2 Ningbo Institute of Materials Technology and Engineering, Chinese Academy of Sciences, Ningbo 315201, China \\ *Corresponding authors (email: fangjf@nimte.ac.cn; clyang@whu.edu.cn)
}

\begin{abstract}
Two star-shaped oligofluorenes with hexakis(fluoren-2-yl)benzene as core are designed and synthesized, namely Tp0 and Tp1. Phosphate groups are attached to the side chain of fluorene units of $\mathrm{Tp} 0$ and $\mathrm{Tp} 1$ and enable them alcohol solubility. Owing to the interfacial dipole formed by the phosphate groups, the work function of ITO reduced effectively. When utilized in the inverted polymer solar cells, the $V_{\text {oc }}$ and FF increased compared with the device without interlayer. PCEs up to 5.30\% and 5.12\% are achieved with $\mathrm{Tp} 0$ and $\mathrm{Tp} 1$ as the interlayers, respectively.
\end{abstract}

Keywords: phosphate group, alcohol soluble, interlayer, polymer solar cells

doi: $10.1360 / \mathrm{N} 032015-00263$ 